\title{
The Continuous Time Random Walk and the Finite Difference Method for the Space Time Fractional Diffusion Equations
}

\author{
Abdel-Rehim EA* \\ Department of Mathematics and Computer Science, Suez Canal University, Egypt
}

Submission: August 28, 2017; Published: October 13, 2017

*Corresponding author: Abdel-Rehim EA, Department of Mathematics and Computer Science, Faculty of Science, Suez Canal University, Ismailia, Egypt, Email: entsarabdelrehim@yahoo.com, entsarabdelrehim@gmail.com

\section{Abstract}

The space fractional diffusion equations are a linear partial pseudo-differential equation with spatial fractional derivatives in space. This equation arises when the motion of the particle is not Brownian and there are extremely long jumps whose length is distributed according to the Lévy long tail $\sim|x|^{-1-\alpha}, 0<\alpha<2$ Some physical, biological and chemical models exhibit a power law with a non integer frequency of order $t^{-\beta}, 0<\beta<1$. In this case, one needs to replace the first order time differential operator by the Caputo time fractional operator. In this paper, I am interested in finding the approximation solution of the space time fractional diffusion equations by using the common finite difference rules beside using the Gruenwald-Letnikove difference scheme for the fractional differential operators. The approximation solution is proved to be conditionally stable. Finally, some numerical examples are given based on this method.

MSC 2010 : Primary 26A33, Secondary 45K05, 60J60, 44A10, 42A38, 60G50, 65N06, 47G30,80-99

Keywords: Diffusion equations; Finite difference; Caputo fractional derivative; Riesz fractional operator; Stationary solutions; Lévy stable distribution; Continuous time random walk

\section{Introduction}

In 1827 an English botanist, Robert Brown, noticed that small particles suspended in fluids perform peculiarly erratic movements. This phenomenon, which can also be observed in gases, is referred to as Brownian motion [1]. It was not until 1905 that Albert Einstein first advanced a satisfactory theory. Einstein considered the case of the free particle, that is, a particle in which no forces other than those due to the molecules of the surrounding medium are acting. He was able to show that the probability density $u(x, t)$ must satisfy the partial differential equation

$$
\frac{\partial u(x, t)}{\partial t}=a \frac{\partial^{2} u(x, t)}{\partial x^{2}},
$$

where $a>0$ is a certain physical constant. This equation is called the diffusion equation or the free diffusion. The conditions imposed on $u$ are

$$
u(x, t) \geq 0, \int_{-\infty}^{\infty} u(x, t) d x=1
$$

The solution $u(x, t)$ of equation (1.1) with the initial condition $u(x, 0)=\delta\left(x-x_{0}\right)$ is well known as the corresponding Green function or the fundamental solution. As soon as the theory for the free particle was established, a natural question arose how it should be modified in order to take into account the outside forces $F(x)$, for example, gravity which acts in the direction of the $\mathrm{x}$-axis and forces the particle to go back to the origin. When the external force is proportional to the distance from the origin, it has shown that equation (1.1) should be replaced by

$$
\frac{\partial u(x, t)}{\partial t}=a \frac{\partial^{2} u(x, t)}{\partial x^{2}}+(b x u(x, t)), a>0, b>0,-\infty<x<\infty, t>0
$$

This partial differential equation describes the elastic diffusive motion of a bounded particle (for example, small pendulum) and being a special case of the general (diffusionconvection equation), see [2-4]. equation (1.2) is a special case from Fokker Planck equation which reads 


$$
\frac{\partial u(x, t)}{\partial t}=L_{f p} u(x, t), a>0, b>0,-\infty<x<\infty, t>0,
$$

Where

$$
L_{f p} u(x, t)=\frac{\partial u^{2}(x, t)}{\partial x^{2}}-\frac{\partial F(x) u(x, t)}{\partial x} .
$$

It is well known that the Ehrenfest Model is mathematically modelled by the Fokker Planck equation. The Fokker-Planck equation (FPE ) is one of the most widely used equations of statistical physics for studying the dynamic behaviour of stochastic differential equations driven by Gaussian noises, see [5-7]. However, it turns out that many other physical phenomena are outside this frame work. Especially solutes that move through fractal media commonly exhibits large deviations from the stochastic processes of Brownian motion and do not require finite velocity. The extension to Lévy stable motion is a straight forward generalization due to the common properties of Lévy stable motion and Brownian motion, but the Lévy flights differ from the regular Brownian motion by the occurrence of extremely long jumps whose length is distributed according to the Lévy long tail $\sim|x|^{-1-\alpha}, 0<\alpha<2$ Many physical, biological, medical, and chemical models exhibits a power law with a non integer frequency of order $t^{-\beta}$, where $0<\beta<1$. for diffusion processes and $0<\beta<2$ for wave propagation. Also in a complex inhomogeneous conducting medium experimental evidence shows that the sound waves propagate with power law of non integer order. $1<\beta<2$

Such processes have a memory. In this paper, I give a review about the approximate, analytical and the continuous time random walk of the space time fractional diffusion processes under the action of constant and linear forces. Therefore, this paper is written in 6 sections. Section 1 is devoted to the introduction. Section 2 is to the important used notations. In section 3, I study the continuous time random walk of the space fractional diffusion equations. In section 4 , the approximate and reversibility of the time fractional Ehrenfest model are discussed. Sections 5 and 6 are devoted to the explicit and implicit approximate solutions of the diffusion equations under the action of a constant force.

\section{Important notations}

In this research papers, I use the space fractional operator $\frac{\partial^{\alpha} u(x, t)}{\partial x^{\alpha}}={ }_{x} D_{0}^{\alpha} u(x, t)$,

which is called the Feller space-fractional differentiation operator of order $\alpha$, where and I adopt the notation introduced by Zaslavsky, who considered it as a power of the positive definitive operator ${ }_{x} D_{0}^{2}=-\frac{d^{2}}{d x^{2}}$, see Oldham \& Spanier [8], Ross \& Miller [9], and recently Gorenflo \& Mainardi [10-13]. This form must not be confused with a power of the first order differential operator ${ }_{x} D_{0}^{1}$. The Fourier transform of ${ }_{x} D_{0}^{\alpha}$ reads

$$
f\left\{D_{0}^{\alpha} \Phi(x) ; k\right\}=-|k|^{\alpha} \hat{\phi}(k), 0<\alpha \leq 2, k \in \mathbb{R},
$$

While

$$
f\left\{\frac{d^{n}}{d x^{n}} \Phi(x) ; k\right\}=-(i k)^{n} \hat{\phi}(k), n \in \mathbb{N}, k \in \mathbb{R}
$$

The operator ${ }_{x} D_{0}^{\alpha}$ is expressed as the negative inverse of the suitable integral operator (Riesz potential) $I_{0}^{\alpha}$ whose symbol is $\left(|k|^{-\alpha}\right)$ and can be written as

$$
{ }_{x} D_{0}^{\alpha}=-I_{0}^{-\alpha}, 0<\alpha \leq 2, \alpha \neq 1,
$$

Where $I_{0}^{-\alpha}$ is the inverse of the symmetric Riesz potential. fractional integral operators $I_{0}^{-\alpha}$ is defined as

$$
I_{0}^{-\alpha} \Phi(x)=c-(\alpha) I_{+}^{\alpha} \Phi(x)+c_{+}(\alpha) I_{-}^{\alpha} \Phi(x), \alpha \neq 1,
$$

where

$$
c-(\alpha)=c_{+}(\alpha)=1 /\left(2 \cos \frac{\alpha \pi}{2}\right)
$$

Therefore, the symmetric Riesz potential can be written as

$$
I_{0}^{-\alpha} \Phi(x)=\frac{1}{2 \cos \frac{\alpha \pi}{2}}\left(I_{+}^{\alpha} \Phi(x)+I_{-}^{\alpha} \Phi(x)\right), 0<\alpha \leq 2, \alpha \neq 1,
$$

Where $I_{ \pm}^{\alpha}$ denote the Riemann-Liouville fractional integral operators, by some people called Weyl integrals. Because of equation (5.3), the approximation solution of this equation are studied at $\alpha=2,0<\alpha<1,1<\alpha<2$ and $\alpha=1$ separately.

\section{Grünwald-Letnikov Scheme for all Values of}

The approximating of ${ }_{x} I_{ \pm}^{-\alpha}$ in the Grünwald-Letnikov scheme for the different values of $\alpha$ is listed below. Firstly for $0<\alpha<1$

$$
I_{ \pm}^{-\alpha} \phi(x)=\frac{1}{h^{\alpha}} \sum_{k=0}^{\infty}(-1)^{k}\left(\begin{array}{l}
\alpha \\
k
\end{array}\right) \phi\left(x_{j} \mp k h\right),
$$

secondly for $1<\alpha \leq 2$

$$
I_{ \pm}^{-\alpha} \phi(x)=\frac{1}{h^{\alpha}} \sum_{k=0}^{\infty}(-1)^{k}\left(\begin{array}{l}
\alpha \\
k
\end{array}\right) \phi\left(x_{j} \mp(k-1) h\right) .
$$

Third for $\alpha=1$, One cannot use the Grünwald-Letnikov discretization of ${ }_{x} D_{0}^{\alpha}$ as $\alpha=1$, because the denominator in equation (5.3). Instead of the Grünwald-Letnikov discretization, I use the discretization used by R. Goreno, who replaced the factor $(-1)^{k}\left(\begin{array}{l}\alpha \\ k\end{array}\right) k \in \mathbb{Z}$, by $\frac{-2}{\pi h}$ for $k=0$ and $\frac{1}{\pi h|k|(|k|+1)}$

\section{Caputo time-fractional derivative}

The time fractional derivative operator of order is defined as

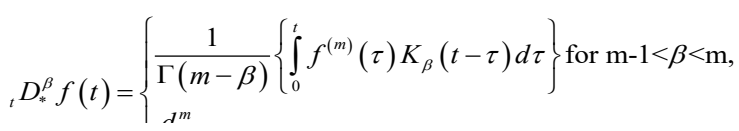

With

$$
K_{\beta}(t-\tau)=\frac{(t-\Gamma)^{\beta+1-m}}{\tau(m-\beta)},
$$

being called the memory function, see [14-18]. This kernel enables us to reflect the memory effects of many physical processes. The Laplace transform of it reads $\mathrm{L}\left\{D_{*}^{\beta} f(t) ; s\right\}=s^{\beta} \tilde{f}(s)-s^{\beta-1} f(0)-f(0) s^{\beta-2}-\ldots-f^{(m-1)}(0) s^{\beta-m}$. 
The descretization of ${ }_{x} D_{0}^{\beta} u(x, t)$ reads

${ }_{\tau} D_{*}^{\beta} y j^{(n+1)}=\sum_{m=0}^{n+1}(-1)^{m}\left(\begin{array}{c}\beta \\ m\end{array}\right) \frac{y_{j}^{(n+1-m)}-y_{j}^{(0)}}{\tau^{\beta}}, 0<\beta \leq 1$.

\section{The continuous time random walk of the diffusion equations}

The space-fractional diffusion equation, [19], reads

$\frac{\partial u(x, t)}{\partial t}=a_{x} D_{0}^{\alpha} u(x, t), 0<\alpha \leq 2, u(x, 0)=\delta(x)$

Where

$\left\{\begin{array}{l}|\theta| \leq \alpha, \quad \text { if } 0<\alpha \leq 1, \\ |\theta| \leq 2-\alpha, \quad \text { if } 1<\alpha \leq 2\end{array}\right.$

$\{|\theta| \leq 2-\alpha$, if $1<\alpha \leq 2$.

This equation has a solution in the Fourier domain, namely $\hat{u}(k, t ; \theta)=\exp \left[-t|k|^{\alpha} e^{\frac{i \theta \pi}{2} \operatorname{sig}(k)}\right], \hat{u}(k, 0)=1$.

The space fractional diffusion with central linear drift reads $\frac{\partial u(\xi, \tau)}{\partial \tau}=b \frac{\partial}{\partial \xi}(\xi u(\xi, \tau))+a_{\xi} D_{0}^{\alpha} u(\xi, \tau), 0<\alpha \leq 2$,

Where $a>0$ and $b>0$. This equation has a solution in the Fourier domain as

$$
\hat{u}(k, t)=\exp \left[-|k|^{\alpha} \frac{a}{b \alpha}\left(1-e^{-\alpha \tau}\right) e^{i \theta \frac{\pi}{2} \operatorname{sig}(k)}\right] .
$$

I use Bilers transformation between the two pairs of the independent variables $(x, t)$ and $(\xi, \tau)$ as

$$
\xi=x(\alpha t+1)^{-1 / \alpha}, \tau=\alpha^{-1} \log (\alpha t+1),(3.6)
$$

Or

$$
x=\xi e^{\tau}, t=\frac{1}{\alpha}\left(e^{\alpha \tau}-1\right) \text {. }
$$

This transformation enables to transfer the solution of the space-fractional diffusion equation to be a solution of the spacefractional Fokker-Planck equation. In [19], I use the Monte Carlo method to simulate the random walk of the discussed model. For this purpose, I generate two random variables $\left(X_{n}, T_{n}\right)$. First we suppose that a particle starts at the origin $\left(x_{0}=0\right)$, and waits a period of time $T_{n}$, at a particular location $\left(x_{n-1}, T_{n}\right)$ before moving instantaneously to the next location with jump width $X_{n}, \forall_{n}=0,1,2, \ldots$ We call $T_{n}$ the waiting times, where $T_{n}=t_{n}-t_{n-1}, t_{n}>t_{n-1}$. The waiting times $T_{n}$ are iid and likewise the jumps $X_{n}$ are iid. Furthermore the waiting times and the jumps are independent of each other. The new position at $t_{n}=t_{n-1}+T_{n}$ which is equivalent to

$$
\begin{aligned}
& t_{0}=0, t_{n}=\sum_{n=1}^{n} T_{n},(3.8) \\
& \text { Is } x_{n}=x_{n-1}+X_{n}, \text { which equals to } \\
& x_{0}=0, x_{n}=\sum_{n=1}^{n} X_{n}, \text { (3.9) }
\end{aligned}
$$

and the particle remains resting at $x=x_{n-1}$ in the time interval $t_{n}<t<t_{n+1}$. For generating the random variable $X_{n}$, i. e. the jump, having the Cauchy or the Gaussian distributions, we use the method of inversion which seems to be simple and most effective. We also use the same method to simulate the random variable T, i. e. the waiting time, having an exponential distribution with mean 1 . For simulating the jump X corresponding stable distribution, $\alpha \in(0,2)$, we use the method given in the book of Janciki [21], in order to simulate the random variable $\mathrm{X}$ having a symmetric and nonsymmetric $\alpha$-stable distribution. Firstly, for the symmetric $\alpha$-stable random variable $\mathrm{X}$, i. e. $\beta=0$ we follow the steps

a) Generate a random variable $V$ uniformly distributed on $-\pi / 2, \pi / 2$ and an exponential random variable $W$ with mean 1 ,

b) Compute

$$
X=\frac{\sin (\alpha V)}{(\cos (V))^{1 / \alpha}}\left(\frac{\cos (V-\alpha V)}{W}\right)^{(1-\alpha) / \alpha} \text {. }
$$

Secondly, for the non-symmetric $\alpha$-stable random variables $\mathrm{X}$ with $\beta^{\prime} \in[-1,1]$, one has to follow the following steps

I. Generate a random variable V uniformly distributed $-\pi / 2, \pi / 2$ on and an exponential random variable $\mathrm{W}$ with mean 1 ,

II. Compute

$X=D_{\alpha, \beta, \cdot} \frac{\sin \left(\alpha\left(V+C_{\alpha, \beta}\right)\right)}{(\cos V)^{1 / \alpha}}\left(\frac{\cos \left(V-\alpha\left(V+C_{\alpha, \beta}\right)\right)}{W}\right)^{(1-\alpha) / \alpha},(3.11)$

Where

$$
\begin{aligned}
C_{\alpha, \beta^{\prime}} & =\frac{\arctan (\beta \tan (\pi \alpha / 2))}{\alpha}, \\
D_{\alpha, \beta^{\prime}} & =\left[\cos \left(\arctan \left(\beta^{\prime} \tan (\tan \pi \alpha / 2)\right)\right)\right]^{(1-\alpha) / \alpha} .
\end{aligned}
$$
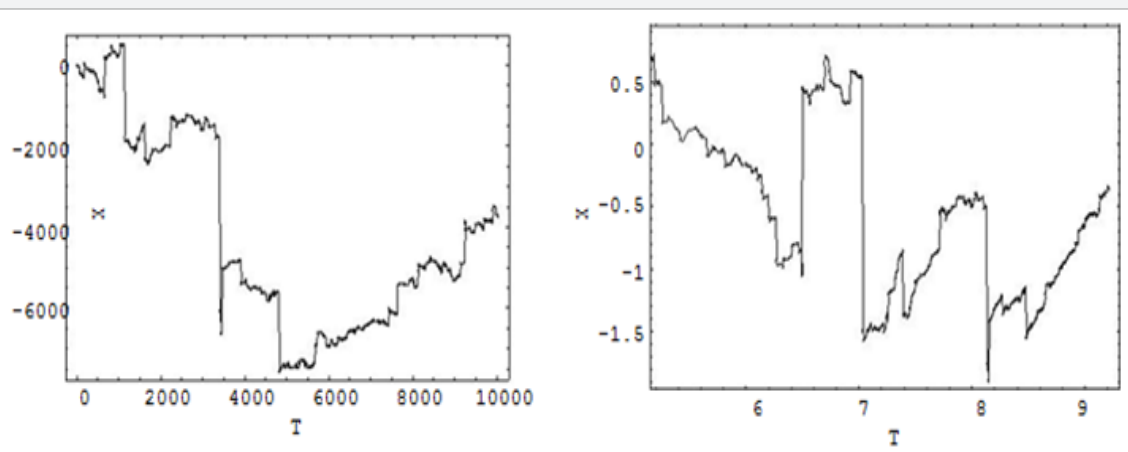

Figure 1: Cauchy, $\alpha=1$, for DE and FPE. 

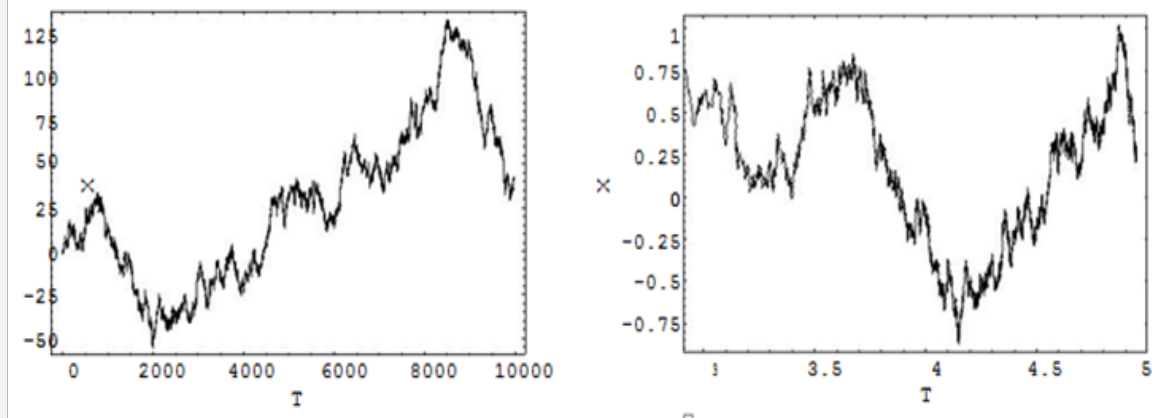

Figure 2: Symmetric Gauss Distribution, $\alpha=2$.
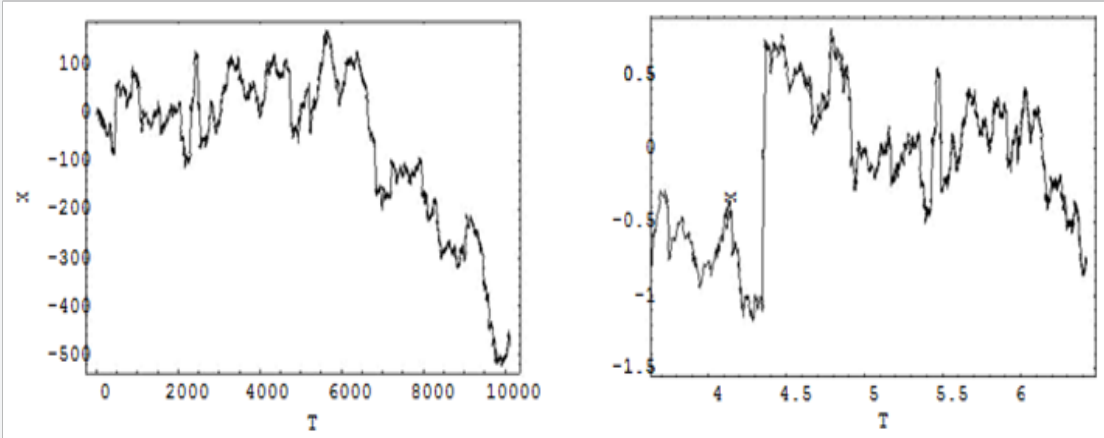

Figure 3: Stable Distribution for $\alpha=1.5, \beta^{\prime}=0$.

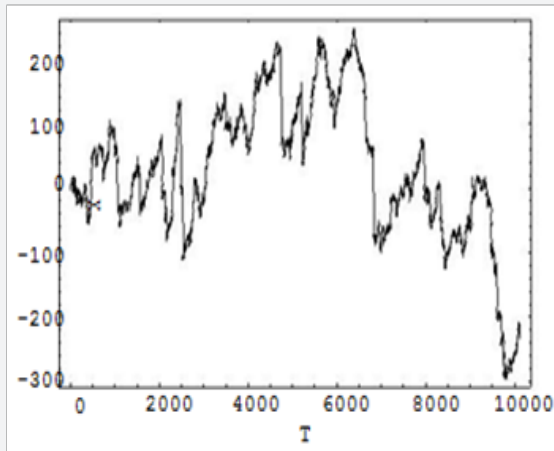

Figure 4: Non Symmetric for $\beta^{\prime}=0.3, \alpha=1.5$.

In the numerical result section, I give some numerical results for the jumps of the diffused particles, see Figures [1-4]. The first column represents the CTRW of the diffusion, DE and the second column represents the CTRW of the diffusion under the action of attractive linear force, FPE.

\section{Time fractional Ehrenfest model}

The Ehrenfest model, classical case, and the time-fractional Ehrenfest model are considered as stochastic processes and are mathematically modelled by the time-fractional diffusion equation under the action of an attractive linear force, equation (3.4. For more information about the classical Ehrenfest model, see [18]. Equation (3.4) for $\alpha=2$ has the solution

$$
u(\zeta, \tau)=p\left(\zeta_{0} ; \zeta, \tau\right)=\frac{1}{\sqrt{2 \pi \frac{a}{b}\left(1-e^{-2 b \tau}\right)}} e \frac{-\left(\zeta-\zeta_{0} e^{-b t}\right)^{2}}{\frac{a}{b}\left(1-e^{-2 b \tau}\right)} .
$$

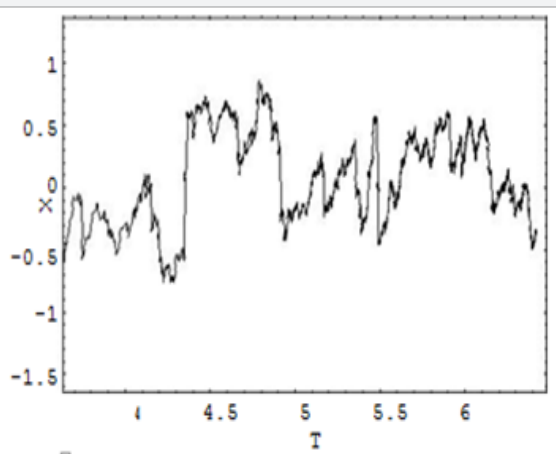

Replace the first order time-derivative by Caputo timefractional derivative of order $\beta$, where $\beta \in(0,1]$ and replace $(\zeta, \tau)$ by simply $(x, t)$. One gets

$$
{ }_{t} D_{*}^{\beta} u(x, t)=a \frac{\partial^{2} u(x, t)}{\partial x^{2}}+\frac{(\partial b x u(x, t))}{\partial x}, a>0, b>0,-\infty<x<\infty
$$

The solution of this equation is getting by using the separation of variables as

$$
u(x, t)=\sum_{n=0}^{\infty} A_{n} E_{\beta}\left(-n t^{\beta}\right) D_{n}(x) e^{-x^{2} / 4},
$$

Where $E_{\beta}\left(-n t^{\beta}\right)$ is the Mittag-Le $\neg$ ffler function and $D_{n}(x)$ is the Weber function. It is worth to say that in [20], I studied the fundamental solution (Green function) of the space-time fractional Ehrenfest model by replacing $\frac{\partial^{2} u(x, t)}{\partial x^{2}}$ in equation (4.1) by ${ }_{x} D_{0}^{\alpha}$. Applying the discretization of the Caputo time fractional operator being given in equation (2.10) besides the common finite difference rule for descretizing $\frac{\partial^{2} u(x, t)}{\partial x^{2}}$ and 
$\frac{\partial b x u(x, t)}{\partial x}$ at equation (4.1), then Solving for $y_{j}^{(n+1)},-R \leq j \leq R$, one

$$
\begin{aligned}
& y_{j}^{(n+1)}=\sum_{k=0}^{n}(-1)^{k}\left(\begin{array}{l}
\beta \\
k
\end{array}\right) y_{j}^{(0)}+\sum_{k=1}^{n}(-1)^{k+1}\left(\begin{array}{l}
\beta \\
k
\end{array}\right) y_{j}^{(n+1-k)} \\
& +y_{j+1}^{(n)}\left[\mu+\frac{\mu h^{2}}{2}(j+1)\right]-2 \mu y_{j}^{(n)}+y_{j-1}^{(n)}\left[\mu-\frac{\mu h^{2}}{2}(j-1)\right] .
\end{aligned}
$$

The discrete scheme for the classical case is put in the matrix form

$$
y^{(n+1)}=P^{T} y^{(n)},
$$

where the matrix $P=p_{i j}$ is defined as

$$
p i j=\left\{\begin{array}{l}
p_{i j}^{(1)}=\frac{2 \mu}{R} \quad \mathrm{j} \geq \mathrm{i}+1, \mathrm{i}=-\mathrm{R}+1, \ldots \ldots, \mathrm{R}-1 \\
p_{i j}^{(2)}=(\beta-2 \mu) \quad \mathrm{j}=\mathrm{i}, \mathrm{i}=-\mathrm{R}, \ldots, \mathrm{R} \\
p_{i j}^{(3)}=\mu\left(2-\frac{2}{R}\right) \quad \mathrm{j}=\mathrm{i}-1, \mathrm{R}+1, \ldots, \mathrm{R}-1 .
\end{array}\right.
$$

Here $\sum_{j=-R}^{R} p i, j=1 \forall i$ that $P^{T}$ means is a stochastic matrix and the classical Ehrenfest forms a Markov chain. While the discrete scheme for the time-fractional Ehrenfest model is put in the matrix form

$$
y^{(n+1)}=Q^{T} y^{(n)}
$$

where the matrix $Q=q i j$ is defined as

$$
q i j=\left\{\begin{array}{l}
q_{i j}^{(1)}=\frac{2 \mu}{R} \quad \mathrm{j} \geq \mathrm{i}+1, \mathrm{i}=-\mathrm{R}+1, \ldots \ldots, \mathrm{R}-1 \\
q_{i j}^{(2)}=(\beta-2 \mu) \mathrm{j}=\mathrm{i}, \mathrm{i}=-\mathrm{R}, \ldots, \mathrm{R} \\
q_{i j}^{(3)}=\mu\left(2-\frac{2}{R}\right) \mathrm{j}=\mathrm{i}-1,-\mathrm{R}+1, \ldots, \mathrm{R}-1 .
\end{array}\right.
$$

Here $\sum^{R} q i, j=\beta \forall i$ That means the matrix $Q^{T}$ is not a stochastic matrix and therefore its process is not a Markov chain. Both matrices can be written as

$$
P=I+\mu H \text { and } Q=\beta I+\mu H,
$$

where $\mathrm{H}$ is a matrix whose its rows are summed to zero. The elements of the stochastic matrix P converge to the binomial distribution at $n \rightarrow \infty$ as

$$
\lim _{x \rightarrow \infty} \mathrm{P}^{n}=\left(\pi_{0} \pi_{1} \ldots \pi_{N}\right),
$$

Where

$$
\pi_{j}=2^{-2 R}\left(\begin{array}{l}
2 R \\
j+R
\end{array}\right),
$$

The stationary row of the matrix $Q$ is getting by using the eigenvector $y^{*}$ with eigenvalue zero of the matrix $\mathrm{H}$. Define $\bar{y}=c y^{*}$, to be the stationary solution of the matrix $\mathrm{Q}$, where $c=1 / \sum_{j=-R}^{R} y_{j}^{*} \cdot$ and $\sum_{-R}^{R} \bar{y}_{j}=1$

\section{Convergence of the Discrete Approximate Solution of both Models}

Define, first the vector $\mathrm{z}$ such that $z^{(n)}=\left(y^{(n)}\right)^{T}$, and the difference vector $d(t)=\left\{d\left(t_{0}\right), d\left(t_{1}\right), d\left(t_{2}\right), \ldots\right\}$, where

$$
d\left(t_{i}\right)=\sum_{j=-R}^{R}\left|z_{j}^{(n)}-\pi_{j}\right|, i=0,1 \ldots
$$

For the Ehrenfest model, $d(t)$ $d(t) \approx c t^{-\gamma}$
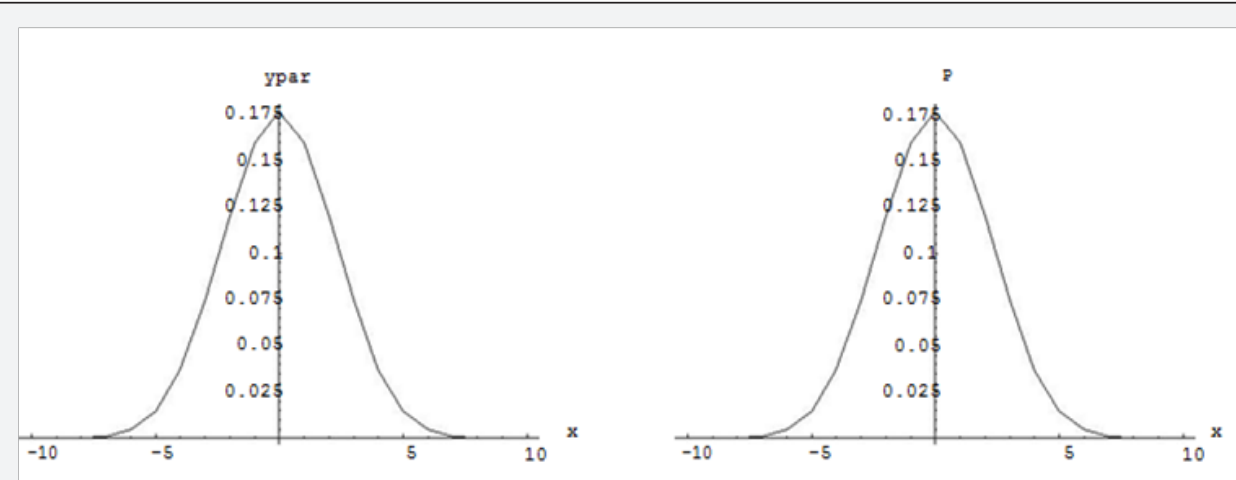

Figure 5: $y$ and $\pi$ for the classical EF.

Where $\omega$ and are constants. The stationary probability vectors and are simulated at Figure [5].

\section{Definition of the reversibility property}

The definition of the reversible process as it is stated at the book of Kelly is as follows: A stochastic process $X(t)$ is said to be reversible if $X\left(t_{1}\right), X\left(t_{2}\right), \ldots, X\left(t_{n}\right)$ has the same distribution as $X\left(\tau-t_{1}\right), X\left(\tau-t_{2}\right), \ldots, X\left(\tau-t_{n}\right)$, for all $X\left(\tau-t_{1}\right), X\left(\tau-t_{2}\right), \ldots ., X\left(\tau-t_{n}\right)$, The balance equation is satisfied for both classical and non classical model where for the classical reads

$$
\pi_{j} p_{j, k}=\pi_{k} p_{k, j}=1, j, k \in \mathbb{N},(4.8)
$$

and for the time-fractional Ehrenfest is

$$
\pi_{j} p_{j, k}=\pi_{k} p_{k, j}=\beta, j, k \in \mathbb{N} \text {, }
$$

although the classical is Ehrenfest is Markov and the non classical is Non-Markov.

\section{The space-time fractional diffusion under the action of a constant force}

The advection dispersion equations, stfade is mathematically models the particle motion at the earth surface. This equation is obtained from equation (1.3) and equation (1.4) by replacing 
$F(x)=-b$, i.e. by a constant force. Here I study its space-time fractional version which reads

$$
\left\{\begin{array}{lc}
{ }_{t} D_{0}^{\beta} u(x, t)=a_{x} D_{0}^{\alpha} u(x, t)-b \frac{\partial}{\partial x} u(x, t) \\
u(x, 0)=\delta(x) & -\mathrm{R} \leq \mathrm{x} \leq \mathrm{R} \\
u(-R, t)=u(R, t)=0 & 0 \leq \mathrm{t} \leq \mathrm{T},
\end{array}\right.
$$

Where $0<\beta \leq 1,0<\alpha \leq 2, \alpha \neq 1$. Here a, and $\mathrm{b}$ are positive constants representing the dispersion coefficient and the average fluid velocity respectively. Here ${ }_{t} D_{*}^{\beta}$ is the Caputo time-fractional operator. I study its space-time fractional version. Namely I investigate its approximate solution by using the explicit finite difference method, see [22]. The Laplace transform of ${ }_{t} D_{*}^{\beta}$ reads

$$
\mathrm{L}\left\{D_{*}^{\beta} f(t) ; s\right\}=s^{\beta} \tilde{f}(s)-s^{\beta-1} f(0) s>0
$$

${ }_{x} D_{0}^{\alpha}$ is the Riesz space-fractional operator of order which in Fourier domain reads

$$
f\left\{D_{0}^{\alpha} \Phi(x) ; k\right\}=-|k|^{\alpha} \hat{\phi}(k), 0<\alpha \leq 2, k \in \mathbb{R},
$$

Riesz Potential: This operator represents the negative inverse of the Riesz Potential $I_{0}^{\alpha}$ whose symbol is $|k|^{\alpha}$, i.e.

${ }_{x} D_{0}^{\alpha}=I_{0}^{\alpha}$,

where the symmetric Riesz Potential operator is defined as

$$
I_{0}^{\alpha} \Phi(x)=c-(\alpha) I_{+}^{\alpha} \Phi(x)+c_{+}(\alpha) I_{-}^{\alpha} \Phi(x), \alpha \neq 1,
$$

Where

$$
c_{+}(\alpha)=c_{-}(\alpha)=1 /\left(2 \cos \frac{\alpha \pi}{2}\right) \text {. }
$$

Because of equation (5.3), the approximation solution of this equation are studied at $\alpha=2,0<\alpha<11<\alpha<2$ and $\alpha=1$ separately. One must distinguish the descretization of ${ }_{h} I_{ \pm}^{-\alpha}$ with respect to the value of $\alpha$, as follows:

$$
{ }_{h} I_{ \pm}^{-\alpha} y j\left(t_{n}\right)=\frac{1}{h^{\alpha}} \sum_{k=0}^{\infty}(-1)^{k}\left(\begin{array}{l}
\alpha \\
k
\end{array}\right) y_{j \mp k}, 0<\alpha \leq 1,
$$

While

$$
{ }_{h} I_{ \pm}^{-\alpha} y j\left(t_{n}\right)=\frac{1}{h^{\alpha}} \sum_{k=0}^{\infty}(-1)^{k}\left(\begin{array}{l}
\alpha \\
k
\end{array}\right) y_{j \pm 1 \mp k}, 0<\alpha \leq 2,
$$

For $\alpha=1$, one has to replace the factor $(-1)^{k}\left(\begin{array}{l}\alpha \\ k\end{array}\right), k \in \mathbb{Z}$, in equations (5.4) and (5.5) by $\frac{-2}{\pi h}$ for $k=0$, and $\frac{1}{\pi h|k|(|k|=1)}$ for $k \neq 1 k \in \mathbb{Z}$ For the numerical calculations, one has to join the descretization of equation (5.4) and equation (5.5) with that of ${ }_{t} D_{*}^{\beta} u(x, t)$ to get the explicit scheme of the space-time fractional advection equation. The descrete scheme of this equation is written in the matrix form

$$
y^{(n+1)}=b_{n} y^{(0)}+\sum_{m=2}^{n} c_{m} y_{i}^{(n+1-m)}+P^{T} \cdot y^{n},
$$

Where

$$
p i j=\left\{\begin{array}{lc}
p_{i j}^{(\mathrm{l})}=\frac{a \mu}{2 \cos \frac{\alpha \pi}{2} R}(-1)^{j-i+1+}\left(\begin{array}{l}
\alpha \\
|j-i|
\end{array}\right) & \mathrm{j} \geq \mathrm{i}+2, \mathrm{i}=-\mathrm{R}+3, \ldots ., \mathrm{R}-3 \\
p_{i j}^{(2)}=\frac{a \mu}{2 \cos \frac{\alpha \pi}{2} R}\left(\begin{array}{l}
\alpha \\
1
\end{array}\right)-\frac{a \mu}{R} & \mathrm{j}=\mathrm{i}+1, \mathrm{i}=-\mathrm{R}, \ldots, \mathrm{R}-3 \\
p_{i j}^{(3)}=\beta-\frac{a \mu}{\cos \frac{\alpha \pi}{2}} & \mathrm{j}=\mathrm{i}=-\mathrm{R},-\mathrm{R}+1, \ldots, \mathrm{R} \\
p_{i j}^{(4)}=\frac{a \mu}{2 \cos \frac{\alpha \pi}{2}}\left(\begin{array}{l}
\alpha \\
1
\end{array}\right)+\frac{a \mu}{R} & \mathrm{j}=\mathrm{i}-1, \mathrm{i}=-\mathrm{R}+1, \ldots, \mathrm{R}-1 \\
p_{i j}^{(s)}=\frac{a \mu}{2 \cos \frac{\alpha \pi}{2}}(-1)^{j-i+1}\left(\begin{array}{l}
\alpha \\
|i-j|
\end{array}\right) & \mathrm{j} \leq \mathrm{i}-2, \mathrm{i}=-\mathrm{R}+3, \ldots \ldots, \mathrm{R}-1
\end{array}\right.
$$
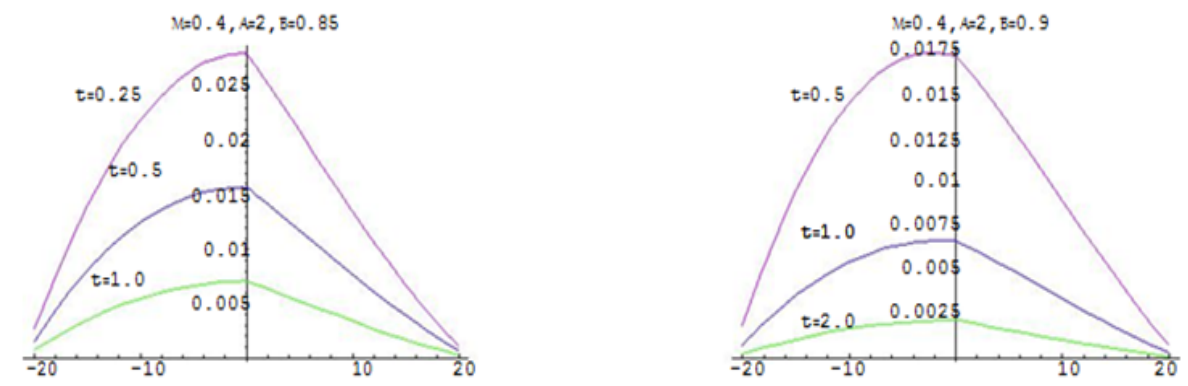

Figure 6: Explicit time evolution of $\mathrm{y}^{(\mathrm{n})}$.
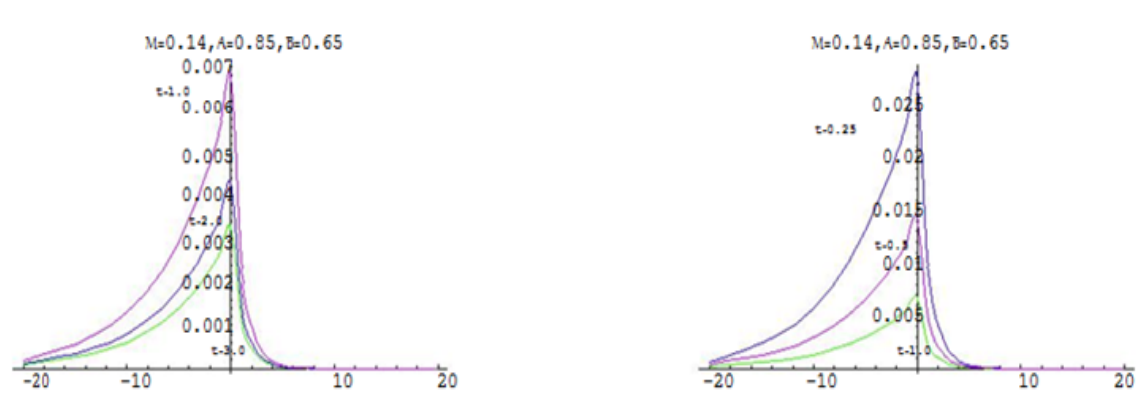

Figure 7: Explicit time evolution of $\mathrm{y}^{(\mathrm{n})}$ 

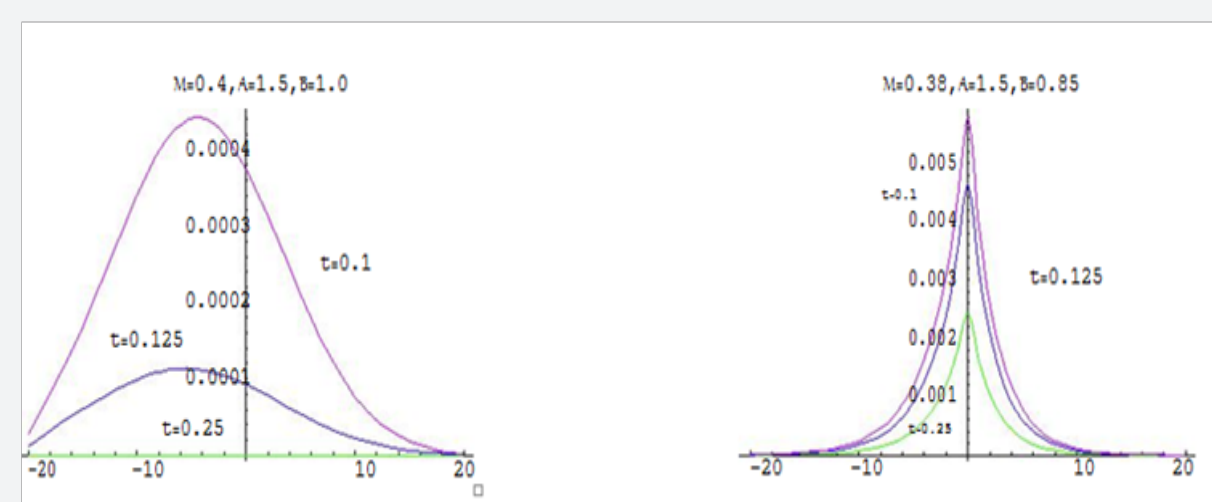

Figure 8: Explicit time evolution of $\mathrm{y}^{(\mathrm{n})}$.
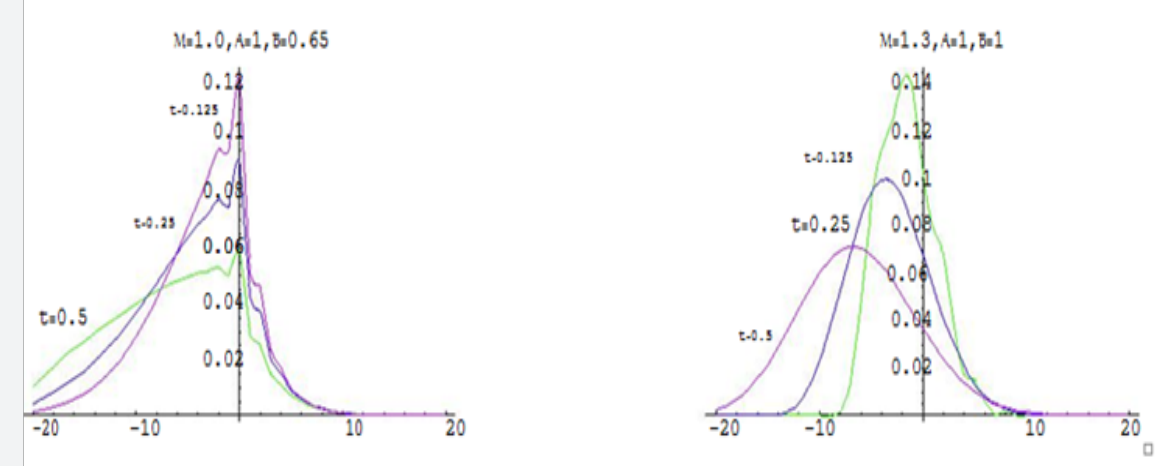

Figure 9: Explicit time evolution of $y^{(n)}$.

Where $|i-j|=k$ and $i, j \in[-R, R]$. I have proved in [22] that the discrete schemes of these model converge, in the Fourier-Laplace domain, to the Fourier-Laplace transforms of the Corresponding fractional differential equations for all values of $\alpha$ and $\beta$. The bath of the diffused particle $y^{(n)}=y\left(t_{n}\right)$ for different values of the time is simulated at Figures [6-8] and . In Figure [9], I simulated the singular case, i.e. as

\section{Implicit difference scheme of the space-time fractional advection diffusion equation}

In this section, I study the implicit difference scheme of the advection diffusion equation (5.1), see [23], by using $\theta-$ the method which is also known as the weighted method. The idea of the method is to replace $y^{(n)}$ in the right hand side of the solved equation (5.6) by

$$
\left(\theta y^{(n+1)}+(1-\theta) y^{(n)}\right)
$$

The implicit method allows to discuss the path of the diffusion in the long run. Therefore, I have numerically compared at this paper the path of the diffusive particles for large values of $\boldsymbol{t}$. The stability analysis of the discrete schemes are discussed and proved for each value of $\alpha$ and $\beta$ according to the VonNeumann Necessary condition for Stability [24]. I have used here the diffusion and drift constants as variables to adjust the value of $\mathrm{R}$ for each values of $\alpha$ and $\beta$. Consequently the value of $\mu$ is also varied. Finally, to simulate the path of the diffused particle for different values of $t, \alpha$ and $\beta$, Figures [10-13].

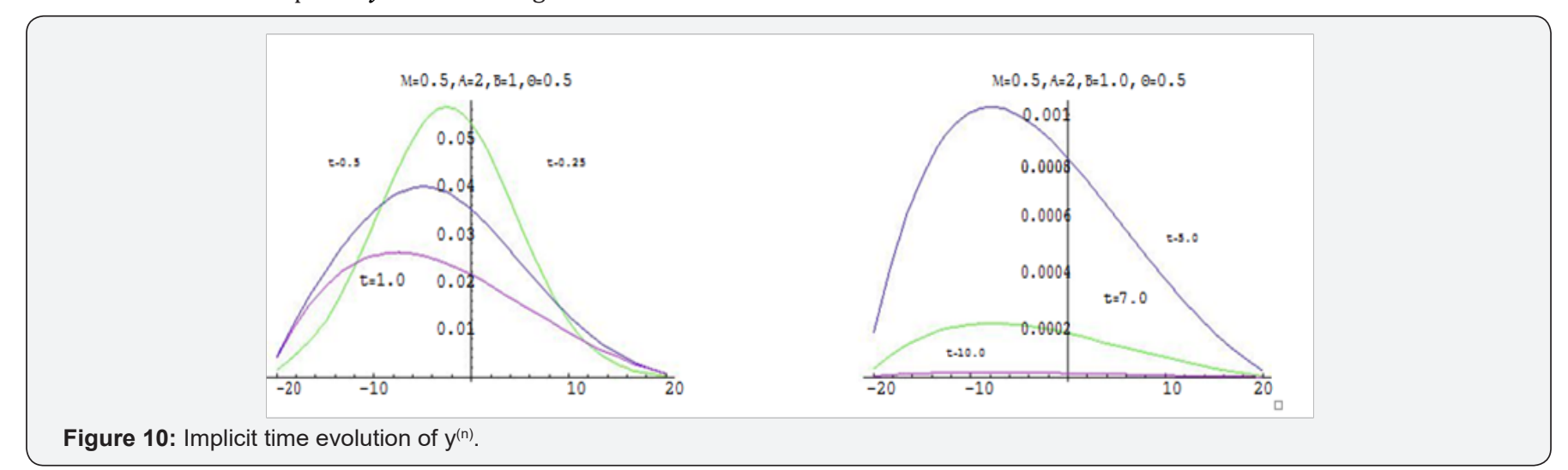



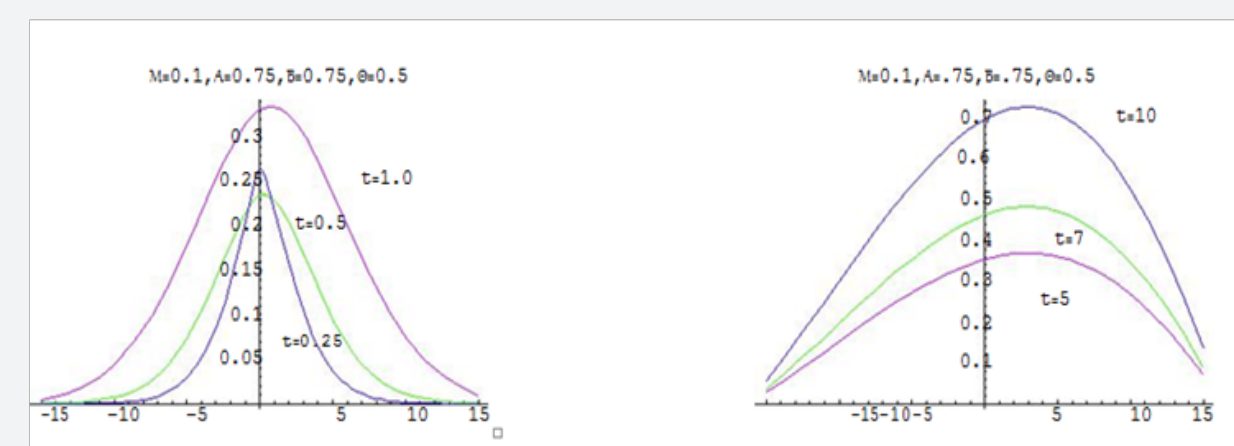

Figure 11: Implicit time evolution of $y^{(n)}$.
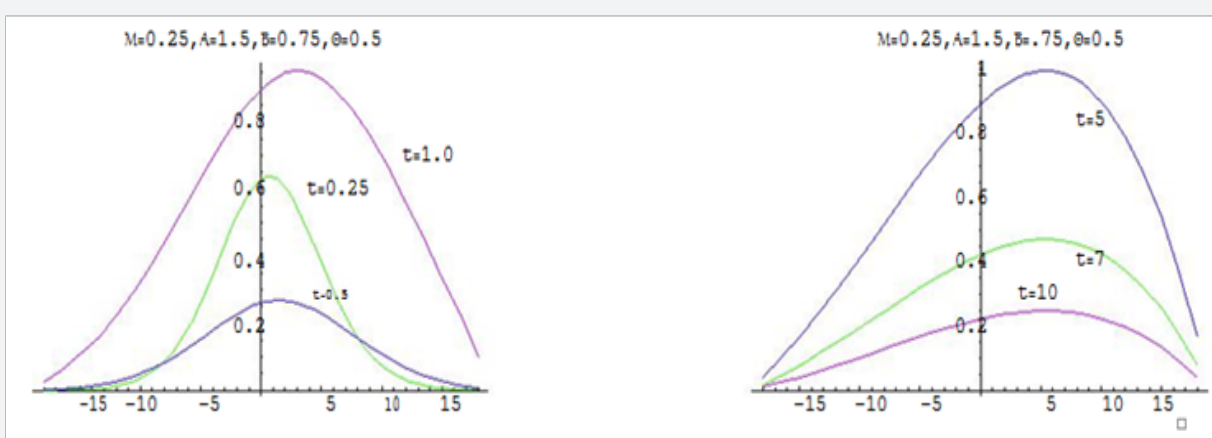

Figure 12: Implicit time evolution of $y^{(n)}$.
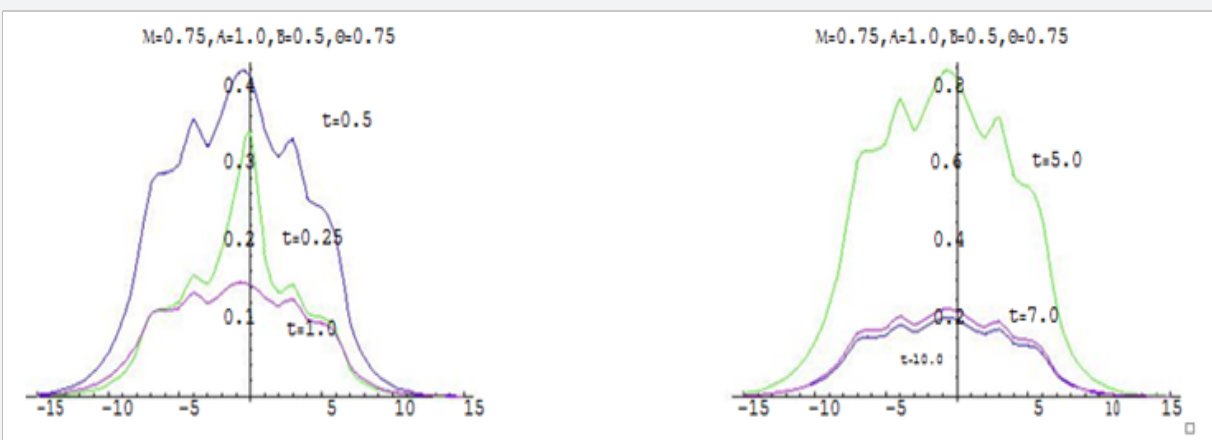

Figure 13: Implicit time evolution of $\mathrm{y}^{(\mathrm{n})}$.

\section{Numerical Result}

Figures [1-13].

\section{References}

1. M Kac (1950) Random walk and the theory of Brownian motion. The American Mathematical Monthly 54(7): 369-391.

2. Uhlenbeck GE, Ornstein LE (1930) On the theory of Brownian motion, Physical Review 36(5): 823-841.

3. Wang MC, Uhlenbeck GE (1956) On the theory of Brownian motion, Reviews of Modern Physics, 17(2-3): 323-342.

4. Smoluchowski, Drei Vortrage über Di usion (1916) Brownsche molekularbe-wegung und koagulation von kolloidteilchen, Zeitschrift für Physic 17: 557-571.

5. Kolwankar KM, Gangal AD (1998) Local fractional Fokker-Planck equation, Physical Review Letters 80(2): 214-217.

6. Metzler R, Barkai E, Klafter J (1998) Anomalous di usion and relax- ation close to thermal equiliubrium: a fractional Fokker-Planck equation approach, Phsical Review Letters 82(18): 3563-3567.

7. Risken H (1989) The Fokker-Planck equation (methods solution and applica-tions) ( $2^{\text {nd }}$ edn.), Springer-Verlag, Berlin, Heidelberg, USA.

8. Oldham KB, Spanier J (1974) The Fractional Calculus, Vol. 3 of Mathematics in Science and Engineering, Academic Press, New York, USA.

9. Miller KS, Ross B (1993) An Introduction to the fractional calculus and fractional differential equations, John Wily and Sons, INC., New York, Chichester, Brisbane, Toronto, Singapore.

10. Gorenflo R, Vivoli A (2003) Fully discrete random walks for the space time fractional diffusion equations, Signal Processing 83(2003): 24112420.

11. Gorenflo R, Mainardi F (1998) Random walk models for spacefractional diffusion processes, Fractional Calculus and Applied Analysis, 1(2): 167-191 
12. Gorenflo R, Mainardi F (1999) Approximation of Lévy- Feller diffusion by random walk, J. for Analysis and its Applications (ZAA) 18: 231-146.

13. Gorenflo R (1997) Fractional calculus: some numerical methods. Fractals and Fractional Calculus in Continuum Mechanics 378: 277 290.

14. Gorenflo R, Mainardi F, Moretti D, Paradisi P (2002) Time-Fractional diffusion: a discrete random walk approach. Nonlinear Dynamics, 29(1-4): 129143

15. Gorenflo R, Abdel-Rehim EA (2005) Discrete models of time-fractional diffusion in a potential well, Fractional Calculus and Applied Analysis 8(2): 173-200.

16. Gorenflo R, Abdel-Rehim EA (2007) Convergence of the GrünwaldLetnikov scheme for time-fractional diffusion. Journal of Computational and Applied Mathematics 205(2): 871-881.

17. Gorenflo R, Abdel-Rehim EA (2008) Simulation of continuous time ran-dom walk of the space-fractional diffusion equations, Journal of Computational and Applied Mathematics 222: 274-285.

18. Abdel-Rehim EA (2009) From the Ehrenfest model to time-fractional stochastic processes. Journal of Computational and Applied Mathematics 233(2): 197-207.
19. Gorenflo R, Abdel-Rehim EA (2008) Simulation of continuous time random walk of the space-fractional diffusion equations, Journal of Computational and Applied Mathematics 222(2): 274-283.

20. Abdel-Rehim EA (2016) Fundamental solutions of the fractional diffusion and the fractional Fokker-Planck equations, Journal of the Egyptian Mathematical Society 24: 337-347.

21. Janicki A (1996) Numerical and statistical approximation of stochastic differential equations with non-gaussian measures, Monograph No. 1 H Steinhaus Center for Stochastic Methods in Science and Technology, Technical University, Worclaw, Poland.

22. Abdel-Rehim EA (2013) Explicit approximation solutions and proof of con-vergence of the space time fractional advection dispersion equations, Scientific Research: Journal of Applied Mathematics 4: 1427-1440.

23. Abdel-Rehim EA (2015) Implicit difference scheme of the space-time frac-tional advection diffusion equation, Journal of fractional calculus \& Ap-plied Analysis, 18(6): 1252-1276.

24. Lax PD, Richtmyer RD (1956) Survey of the stability of linear finite difference equations, Communication on Pure and Applied Mathematics 9(3): 267-293.

\section{Your next submission with Juniper Publishers}

will reach you the below assets

- Quality Editorial service

- Swift Peer Review

- Reprints availability

- E-prints Service

- Manuscript Podcast for convenient understanding

- Global attainment for your research

- Manuscript accessibility in different formats

( Pdf, E-pub, Full Text, Audio)

- Unceasing customer service

Track the below URL for one-step submission https://juniperpublishers.com/online-submission.php 